\title{
DISTANCE 2-DOMINATION IN PRISMS OF GRAPHS
}

\author{
Ferran Hurtado, Mercè Mora ${ }^{1}$ \\ Universitat Politècnica de Catalunya \\ Barcelona, Spain \\ e-mail: merce.mora@upc.edu \\ EDUARDO RIVERA-CAMPO ${ }^{2}$ \\ Universidad Autónoma Metropolitana-Iztapalapa \\ Mexico \\ e-mail: erc@xanum.uam.mx \\ AND \\ Rita ZUAZUA ${ }^{3}$ \\ Universidad Nacional Autónoma de México \\ Mexico \\ e-mail: ritazuazua@ciencias.unam.mx
}

\section{Dedication}

Ferran Hurtado passed away a few months after Eduardo Rivera-Campo and Rita Zuazua visited him and Mercè Mora in Barcelona, where most of this research was made. We all dedicate this final version to Ferran's memory.

\begin{abstract}
A set of vertices $D$ of a graph $G$ is a distance 2-dominating set of $G$ if the distance between each vertex $u \in(V(G)-D)$ and $D$ is at most two. Let $\gamma_{2}(G)$ denote the size of a smallest distance 2-dominating set of $G$. For any permutation $\pi$ of the vertex set of $G$, the prism of $G$ with respect to $\pi$ is the graph $\pi G$ obtained from $G$ and a copy $G^{\prime}$ of $G$ by joining $u \in V(G)$ with $v^{\prime} \in V\left(G^{\prime}\right)$ if and only if $v^{\prime}=\pi(u)$. If $\gamma_{2}(\pi G)=\gamma_{2}(G)$ for any permutation
\end{abstract}

\footnotetext{
${ }^{1}$ Research partially supported by projects MTM2015-63791-R (MINECO/FEDER) and Gen. Cat. DGR 2014SGR46.

${ }^{2}$ Research partially supported by project 178910 , Conacyt, México.

${ }^{3}$ Research partially supported by PAPIIT-IN114415, UNAM.
} 
$\pi$ of $V(G)$, then $G$ is called a universal $\gamma_{2}$-fixer. In this work we characterize the cycles and paths that are universal $\gamma_{2}$-fixers.

Keywords: distance 2-dominating set, prisms of graphs, universal fixer.

2010 Mathematics Subject Classification: 05C69.

\section{REFERENCES}

[1] C.M. Mynhardt and Z. Xu, Domination in prisms of graphs: universal fixers, Util. Math. 78 (2009) 185-201.

[2] M. Lemańska and R. Zuazua, Convex universal fixers, Discuss. Math. Graph Theory 32 (2012) 807-812. doi:10.7151/dmgt.1631

[3] A. Meir and J.W. Moon, Relations between packing and covering number of a tree, Pacific J. Math. 61 (1975) 225-233. doi:10.2140/pjm.1975.61.225

[4] C.M. Mynhardt and M. Schurch, Paired domination in prisms of graphs, Discus. Math. Graph Theory 31 (2011) 5-23.

doi:10.7151/dmgt.1526

[5] K. Wash, Edgeless graphs are the only universal fixers, Czechoslovak Math. J. 64 (2014) 833-843.

doi:10.1007/s10587-014-0136-3

Received 27 November 2015

Revised 2 June 2016

Accepted 2 June 2016 